\title{
Blind Identification Using Higher Order Statistics and Eigendecomposition
}

$\begin{array}{lrcc}\text { Nonmember } & \text { Ling } & \text { CHEN } & \text { (University of Osaka Prefecture) } \\ \text { Nonmember } & \text { Hiroji } & \text { KUSAKA } & \text { (University of Osaka Prefecture) } \\ \text { Member } & \text { Masanobu KOMINAMI } & \text { (University of Osaka Prefecture) } \\ \text { Nonmember } & \text { Qingyie } & \text { YIN } & \text { (Xian Jiantong University) }\end{array}$

\section{Summary}

In this paper we develop a new approach to identify noncausal AR models driven by non-Gaussian i.i.d input. Under a few moderate assumptions, we derive the necessary and sufficient conditions of rebuilding the parameters of the AR models from 2nd and higher order statistics. We show that the AR model parameters are directly related to the solution of an eigenproblem. Based on the result, we present a method of AR model identification, applying eigenvector computation. The unique solution of AR parameters is guaranteed up to sign and linear phase ambiguity. Model order determination is not crucial in the method. If the order is overestimated, several equivalent AR models with different linear phases will be obtained.

Keyword: Higher order statistics, Non-minimum phase, Blind identification, Noncausal AR model

\section{Introduction}

The blind identification of non-minimum phase systems has received extensive attention in recent years. Higher order statistics provide us a powerful tool to reconstruct non-minimum phase systems only from observing the output, if the systems are driven by nonGaussian, independent, identically distributed (i.i.d) input. Many successful methods of identifying MA, $A R$ and ARMA models have been developed based on higher order cumulants. Two excellent overviews can be found in [1][2].

The correlation based approach only solves the spectrally equivalent minimum phase systems because 2ndorder statistics do not carry complete phase information. For AR models, Yule-Walker equation is solvable only if the systems have causal impulse responses, i.e. they are minimum phase systems. To handle nonminimum phase, the systems are often considered as MA ${ }^{[4][5]}$ or ARMA ${ }^{[8][7]}$ models that have causal impulse responses. However it has been found very convenient to specify non-minimum phase processes as noncausal
AR models in certain applications such as spatial signal processing, astronomical data processing and blind equalization.

As pointed out in [2], the identification of nonminimum phase systems is classified into three categories: exhaustive search, nonlinear optimization, and linear algebraic solution. Since we are more interested in linear approach, the exhaustive search and optimization methods will not be taken into our consideration. For noncausal AR models, most methods essentially convert the problem to the related MA model identification ${ }^{[2][3]}$ [7][9]. For example in [3], the identification of noncausal AR models is studied, where an equivalent MA model is employed to make the normal equation solvable. Consequently the result is sensitive to the choice of the MA model order and may suffer greatly from the poles located near the unit circle. Other methods that essentially convert the problem to MA model identification can be found in [7].

In our study, we propose a linear approach of identifying noncausal AR models. First we present the conditions of uniquely determining AR parameters, using 
both correlation lags and higher order cumulant samples. We find that the AR model coefficients are directly related with a typical eigenproblem. Based on the result, eigendecomposition is applied and the AR coefficients are obtained from the proper choice of the eigenvectors. Singular value decomposition(SVD) may also be used as a more general method. Order determination is not crucial in the method. The overestimated model order simply leads to linear phase ambigurity among the solutions yielded by the proposed algorithm. The method is valid when some poles are located near the unit circle. But it does suffer from the zeros near $|z|=1$, because the data are not suitable to fit AR models in this situation.

The paper is organized as follow. In Section 2, the problem is formulated under some moderate constraint. An indirect form of the correlation function is introduced to cope with additive Gaussian noise. In Section 3 , we give the proof of uniquely determining AR coefficients, and then present an algorithm of AR model identification, using eigenvalue analysis. The simulation results of this approach are demonstrated in Section 4.

\section{Problem Formulation}

$\langle\mathbf{2} \cdot \mathbf{1}\rangle$ Blind identification Consider a linear system that has impulse response $h(t)$, the output of the system is given by

$$
y(t)=\sum_{i=-\infty}^{\infty} h(t-i) x(i)
$$

where the input $x(t)$ is a non-Gaussian i.i.d sequence with zero mean. Now the system is specified by an AR model as

$$
\begin{aligned}
H(z) & =\frac{A}{\prod_{i=1}^{p}\left(1-u_{i} z\right) \prod_{i=1}^{q}\left(1-v_{i} z^{-1}\right)} \\
& =\frac{1}{\sum_{i=-p}^{q} a(i) z^{-i}} \cdots \cdots \ldots \ldots \ldots
\end{aligned}
$$

In the expression, we assume $\left|u_{i}\right|<1$ and $\left|v_{i}\right|<1$, so that $\prod_{i=1}^{p}\left(1-u_{i} z\right)$ is the maximum phase factor with anti-causal impulse response and $\prod_{i=1}^{q}\left(1-v_{i} z^{-1}\right)$ is the minimum phase factor with causal response. In the time domain (2) is equivalent to

$$
\sum_{i=-p}^{q} a(i) y(t-i)=x(t)
$$

Thus the problem of blind identification is described as reconstructing the coefficient $\{a(i)\}$ from observing $y(t)$ when $x(t)$ is not accessible. Since neither the linear phase (delay) nor the sign of $H(z)$ can be measured from $y(t)$, we formulate the problem in an alternative way.

For a given set of $\{a(i)\}_{i=-p}^{q}$, we define a residual function as

$$
f(t)=\sum_{i=-p}^{q} a(i) h(t-i) \cdots \cdots \cdots \cdots \cdots \cdots \cdots \cdot(4)
$$

Here $\{a(i)\}$ will be considered as the solution of the $A R$ model $H(z)$ if $f(t)$ has only one nonzero, i.e.

$$
f(t)= \begin{cases} \pm 1 & \text { if } t=d \\ 0 & \text { otherwise }\end{cases}
$$

where $d$ is an arbitrary integer. If the model order is overdetermined, i.e. $a(-p)=0$ or $a(q)=0$, another AR model whose parameters are specified by shifting $\{a(i)\}_{i=-p}^{q}$ also satisfies (5). In this case the two models are viewed identically from the perspective of blind identification. In other words, $H(z)$ and $z^{r} H(z)$ are equivalent in our study. The sign ambignity in (5) does not make much sense and can be ignored.

The idea behind the formulation is that we use an FIR filter that has coefficients $\{a(i)\}$ to compensate $H(z)$. The the identification of the unknown system $H(z)$ is achieved if a delayed version of $x(t)$ is obtained from the filter.

$\langle 2 \cdot 2\rangle \quad$ Higher order statistics The explicit definition of higher order cumulants is given in [2]. For the zero-mean stochastic process $y(t)$, the 3rd-order cumulant takes identical form to its 3rd-order moment, i.e.

$$
C_{3 y}\left(\tau_{1}, \tau_{2}\right)=E\left\{y(t) y\left(t+\tau_{1}\right) y\left(t+\tau_{2}\right)\right\} \cdots \cdots(6)
$$

In the case of the linear system $H(z)$ driven by $x(t)$, it is easy to show that

$$
C_{3 y}\left(\tau_{1}, \tau_{2}\right)=\gamma_{3 x} \sum_{i=-\infty}^{\infty} h(i) h\left(i+\tau_{1}\right) h\left(i+\tau_{2}\right) \cdots(7)
$$

where $\gamma_{3 x}=E\left\{x^{3}(t)\right\}$ is the skewness of $x(t)$.

In our study we will use 3rd-order cumulant for simplicity because the obtained result can be easily extended to 4 th-order cumulant.

An important property of higher order cumulants is that they are insensitive to additive Gaussian noise ${ }^{[2]}$

The fact that relation (7) remains valid even if $y(t)$ is corrupted by Gaussian noise allows us to ignore the presence of Gaussian noise theoretically, if the estimates are based on higher order statistics. But in our method, both 3rd-order cumulant $C_{3 y}\left(\tau_{1}, \tau_{2}\right)$ and correlation $r(\tau)=E[y(t) y(t+\tau)]$ are used. To cope with 
Gaussian noise, we can make use of an indirect form of correlation function given by

$$
\begin{aligned}
r(\tau) & =\frac{\sigma_{x}^{2}}{\gamma_{3 x} \sum_{n=-\infty}^{\infty} h(n)} \sum_{n=-\infty}^{\infty} C_{3 y}(\tau, n) \\
& \approx \alpha \sum_{n=-L}^{L} C_{3 y}(\tau, n)
\end{aligned}
$$

where $L$ must be large enough and $\alpha$ is a constant since $\sum_{n=-\infty}^{\infty} h(n) \neq 0$ for AR models. We need not worry about the unknown constant $\alpha$ too much because its effect is similar to that of unknown $\gamma_{3 x}$

Therefore with higher order cumulant the following assumptions are made reasonable for further analysis:

1. The AR model is free from additive noise;

2. $x(t)$ is non-Gaussian i.i.d and $E\{x(t)\}=0, \gamma_{3 x} \neq 0$; furthermore we assume $E\left\{x^{2}(t)\right\}=1$ instead of $h(0)=1$.

3. $H(z)$ does not have zeros and poles on the unit circle.

\section{Identifying Noncausal AR Models}

The blind identification of non-minimum phase AR models is described as finding the coefficients $\{a(i)\}$ so that the residual function $f(t)$ defined in (4) has only one nonzero. Now we present the following theorem.

Theorem 1 For a given coefficient set $\{a(i)\}_{i=-p}^{q}$, the residual function $f(t)$ has only one nonzero if and only if the following conditions are satisfied for all $m$ and some $k$,

$$
\begin{array}{r}
\sum_{n=-p}^{q} C_{3 y}(m-k, m-n) a(n)=\lambda_{k} \sum_{n=-p}^{q} r(m-n) a(n) \cdots(8) \\
\lambda_{k}=\gamma_{3 x} \sum_{n=-p}^{q} r(k-n) a(n) \neq 0 \cdots \cdots \cdots \cdot(9) \\
\sum_{n=-p}^{q} \sum_{l=-p}^{q} r(l-n) a(n) a(l)=1 \cdots \cdots \cdots \cdot(10)
\end{array}
$$

Proof: First we prove that (8)-(10) can be obtained if $\{a(i)\}$ are the true AR coefficients.

Since $\{a(i)\}$ are the true coefficients, relation (3) holds. Multiply (3) by $y(t-m)$ and take average, we have

$$
\sum_{n=-p}^{q} r(m-n) a(n)=h(-m) \cdots \cdots \cdots \cdots
$$

This relation can be viewed as Yule-Walker equation for noncausal AR models. Similarly, multiply (3) by $y(t-k) y(t-m)$ and recall $(7)$, we get

$$
\sum_{n=-p}^{q} C_{3 y}(m-k, m-n) a(n)=\gamma_{3 x} h(-k) h(-m)(12)
$$

Let $\lambda_{k}=\gamma_{3 x} h(-k)$ and use (11), we obtain (8) and (9). It is not difficult to demonstrate that for the AR model $(2), h(t)$ can not be equal to zero for all $t \in[-p, q]$. Thus there exists $-k \in[-p, q]$ so that $\lambda_{k}=\gamma_{3 x} h(-k) \neq$ 0 . Relation (10) is obvious because

$$
\sum_{n=-p}^{q} \sum_{l=-p}^{q} r(l-n) a(n) a(l)=E\left\{x^{2}(t)\right\}=1
$$

Up to now we proved that (8)-(10) are necessary conditions if $\{a(i)\}$ are the true AR parameters. Now we will show they are also sufficient for recovering the AR model.

Let us examine the residual function $f(t)$ defined in (4) when $\{a(i)\}$ satisfies (8)-(10). From $r(\tau)=$ $\sum_{i=-\infty}^{\infty} h(i) h(i+\tau)$, it can be found that (9) and (10) are equivalent to

$$
\lambda_{k}=\gamma_{3 x} \sum_{i=-\infty}^{\infty} h(i-k) f(i) \neq 0 \quad \ldots \ldots \ldots \ldots
$$

and

$$
\sum_{i=-\infty}^{\infty} f^{2}(i)=1
$$

respectively. Also we get

$$
\sum_{n=-p}^{q} r(m-n) a(n)=\sum_{i=-\infty}^{\infty} h(i-m) f(i) \cdots
$$

Substitute (7) and (15) into (8), we can rewrite (8) as

$$
\sum_{i=-\infty}^{\infty} h(i-m)\left[\gamma_{3 x} h(i-k)-\lambda_{k}\right] f(i)=0 \cdots
$$

Since (16) holds for all $m$ and $H(z)$ does not have zeros on $|z|=1$, it must be

$$
\left[\gamma_{3 x} h(i-k)-\lambda_{k}\right] f(i)=0 \quad \text { for all } i \cdots \cdots
$$

Take the sum over index $i$ and use (13), we find

$$
\lambda_{k}\left[1-\sum_{i=-\infty}^{\infty} f(i)\right]=0
$$

Since $\lambda_{k} \neq 0$, it must be

$$
\sum_{i=-\infty}^{\infty} f(i)=1 \ldots \ldots \ldots \ldots \ldots \ldots \ldots \ldots \ldots \ldots
$$

The function $f(t)$ satisfies both (14) and (19) if and only if it has one nonzero, or equivalently $\{a(i)\}$ represents the true AR model up to a linear phase. The above deduction is valid if $a(-p)$ or $a(q)$ is equal to zero. Thus the theorem holds for all the equivalent AR models if the model order is overdetermined.

Now we present an algorithm based on the theorem. In fact the model order $(p, q)$ is unknown. But it is 
possible for us to choose $P \geq p$ and $Q \geq q$. Let $m=$ $-P, \cdots, Q$, we rewrite (8)-(10) in the matrix forms of

$$
\begin{aligned}
& C_{k} \boldsymbol{a}=\lambda_{k} \boldsymbol{r} \boldsymbol{a} \\
& \lambda_{k}=\gamma_{3 x} \boldsymbol{I}_{k}^{T} \boldsymbol{r} \boldsymbol{a} \neq 0 \\
& \boldsymbol{a}^{T} \boldsymbol{r} \boldsymbol{a}=1 \cdots
\end{aligned}
$$

where the entries of matrices and vectors are

$$
\begin{aligned}
C_{k}(m, n) & =C_{3 y}(m-k, m-n) \quad m, n=-P, \cdots, Q \\
r(m, n) & =r(m-n) \\
a^{T} & =(a(-P), a(-P+1), \cdots, a(Q)) \\
\boldsymbol{I}_{k}{ }^{T} & =(0, \cdots, 1, \cdots, 0) \quad(1 \text { at } k \text { th })
\end{aligned}
$$

It can be observed that $C_{k}$ and $\boldsymbol{r}$ are symmetric and $\boldsymbol{r}$ is always positive definite. Therefore Cholesky decomposition can be applied to $r$, i.e. the correlation matrix is decomposed as $\boldsymbol{r}=\boldsymbol{L} \boldsymbol{L}^{T}$, where $\boldsymbol{L}$ is a lower triangular matrix and $\left(\boldsymbol{L}^{T}\right)^{-1}=\left(\boldsymbol{L}^{-1}\right)^{T}$. Thus the conditions (20)-(22) are rewritten as

$$
\begin{aligned}
& P_{k} \boldsymbol{v}=\lambda_{k} \boldsymbol{v} \ldots \\
& \lambda_{k}=\gamma_{3 x} \boldsymbol{I}_{k}^{T} \boldsymbol{L} \boldsymbol{v} \neq 0 \\
& \boldsymbol{v}^{T} \boldsymbol{v}=1 \ldots \ldots
\end{aligned}
$$

where $\boldsymbol{P}_{k}=\boldsymbol{L}^{-1} \boldsymbol{C}_{k}\left(\boldsymbol{L}^{-1}\right)^{T}$ is also symmetric and $\boldsymbol{v}=\boldsymbol{L}^{T} \boldsymbol{a}$

It is clear to see that $v$ is the normalized eigenvector belonging to the eigenvalue $\lambda_{k}$. From eigenvalue decomposition we can obtain $P+Q+1$ eigenvectors $\left\{\boldsymbol{v}_{k}^{(i)}\right\}_{i=0}^{P+Q}$, then we have to decide which one among them associates the true solution of the AR model. If $\gamma_{3 x}$ is known, we can do that by testing which one satisfies (24). Unfortunately, (24) will hold only when the true statistics of $y(t)$ are given. In practice we can only choose the eigenvalue-eigenvector pair which is most likely to fit the relation. For this purpose, we define a criterion as

$$
\varepsilon=\frac{\left|\lambda^{(i)}-\gamma_{3 x} \boldsymbol{I}_{k}^{T} \boldsymbol{L} \boldsymbol{v}_{k}^{(i)}\right|}{\left|\lambda^{(i)}\right|+\left|\gamma_{3 x} \boldsymbol{I}_{k}^{T} \boldsymbol{L} \boldsymbol{v}_{k}^{(i)}\right|}
$$

Among $P+Q+1$ eigenvectors we chose the one which minimizes $\varepsilon$ as the desired solution. The criterion does not guarantee the correct choice especially when the estimated cumulant is unreliable. However we find that it gives reasonably good results if more than 1000 samples are used to estimate the cumulants. (e.g. thirteen incorrect choices were made in 500 Monte Carlo runs when 1024 samples were used; and six failures were found in case of 4096 data samples.)

If $\gamma_{3 x}$ is unknown, the proper choice of eigenvectors can be made by comparing the $P+Q+1$ possible solutions. When the model order is overestimated, i.e $P>p$

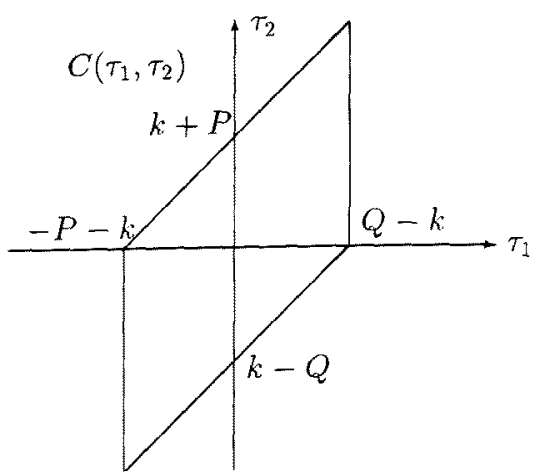

Figure 1 The region where the 3rd-order cumulant samples are used in identification

or $Q>q$, totally $(P+Q)-(p+q)+1$ desired eigenvectors that represent the same number of equivalent $A R$ models will be obtained from the eigenvalue decomposition. The equivalent AR models are distinguishable, because they must be all similar to each other up to a shifted index. From the simulations it was found that the correct solutions can be determined if more 1000 samples are available to estimate the cumulants.

The free parameter $k$ shifts the region where cumulant samples are used by the algorithm (see Fig.1). It is reasonable to let $k=0$. Theoretically, the algorithm may fail if $h(-k)=0$. By overestimating the model order, we can hardly expect that it happens for all the equivalent AR models unless $h(t)$ has many sequential zeros in $t \in[-p, q]$, where $(p, q)$ is the true mode order. For AR models, the impulse responses rarely have many sequential zeros in the origin.

If the input $x(t)$ admits a symmetric distribution, we have to use 4th-order cumulant, because the odd order cumulants are all identical to zero. The definition of 4th-order cumulant in our study is given by

$$
\begin{aligned}
C_{4 y}\left(\tau_{1}, \tau_{2}\right)= & E\left[y^{2}(t) y\left(t+\tau_{1}\right) y\left(t+\tau_{2}\right)\right] \\
& -2 r\left(\tau_{1}\right) r\left(\tau_{2}\right)-r(0) r\left(\tau_{1}-\tau_{2}\right)
\end{aligned}
$$

The condition for identifying noncausal AR models can be extended to the 4th-order case as follows

$$
\begin{gathered}
\sum_{n=-p}^{q} C_{4 y}(k-m, k-n) a(n)=\lambda_{k} \sum_{n=-p}^{q} r(m-n) a(n) \\
\lambda_{k}=\gamma_{4 x}\left(\sum_{n=-p}^{q} r(k-n) a(n)\right)^{2} \neq 0 \\
\sum_{n=-p}^{q} \sum_{l=-p}^{q} r(l-n) a(n) a(l)=1
\end{gathered}
$$

where $\gamma_{4 x}=E\left[x^{4}(t)\right]-3\left\{E\left[x^{2}(t)\right]\right\}^{2}$. The same algorithm can be applied to the formulations. 


\section{Simulations}

In the simulations we use the presented algorithm to identify the noncausal AR model specified by

$$
\begin{aligned}
H_{1}(z) & =\frac{1}{(1+0.3 z)\left(1-0.6 z^{-1}+0.25 z^{-2}\right)} \\
& =\frac{1}{0.3 z+0.820-0.525 z^{-1}+0.25 z^{-2}}
\end{aligned}
$$

Since $H_{1}(z)$ is not physically realizable, the truncated impulse response $h_{t r}(t)$ is employed to approximate $h(t)$. i.e.

$$
h_{t r}(t)= \begin{cases}h(t) & -L_{1} \leq t \leq L_{2} \\ 0 & \text { otherwise }\end{cases}
$$

where $L_{1}$ and $L_{2}$ are chosen as large enough so that the algorithm yields almost perfect identification of $H_{1}(z)$ when the true statistics calculated from $h_{t r}(t)$ are given.

The zero-mean exponentially distributed i.i.d input is generated with $\sigma_{x}=1$ and $\gamma_{3 x}=2$. The correlation and 3rd-order cumulant are estimated from the output samples with data segment length of $N=1024$ and $N=4096$ respectively. The order of the AR model is predetermined as $P=Q=2$. Table 1 shows the mean values and standard deviations of estimated AR coefficients from 500 Monte Carlo runs. Two solutions are obtained. The second one is ap-

\begin{tabular}{|c|c|c|}
\hline \multirow{2}{*}{$\begin{array}{l}\text { True } \\
\text { parameter }\end{array}$} & \multicolumn{2}{|c|}{$N=1024$ (mean \pm std.dev) } \\
\hline & Solution I & Solution II \\
\hline$a(-2)=0.000$ & $-0.003 \pm 0.0488$ & $0.300 \pm 0.0572$ \\
\hline$a(-1)=0.300$ & $0.292 \pm 0.0434$ & $0.812 \pm 0.0530$ \\
\hline$a(0)=0.820$ & $0.802 \pm 0.0947$ & $-0.514 \pm 0.0402$ \\
\hline$a(1)=-0.525$ & $-0.522 \pm 0.1464$ & $0.250 \pm 0.0346$ \\
\hline$a(2)=$ & $0.259 \pm 0.0824$ & $0.002 \pm 0.0494$ \\
\hline \multirow{2}{*}{$\begin{array}{l}\text { true } \\
\text { parameter }\end{array}$} & \multicolumn{2}{|c|}{$N=4096$ (mean \pm std.dev) } \\
\hline & Solution I & Solution II \\
\hline$a(-2)=0.000$ & $0.000 \pm 0.0197$ & $0.302 \pm 0.0275$ \\
\hline$a(-1)=0.300$ & $0.300 \pm 0.0175$ & $0.819 \pm 0.0261$ \\
\hline$a(0)=0.820$ & $0.818 \pm 0.0215$ & $-0.524 \pm 0.0207$ \\
\hline$a(1)=-0.525$ & $-0.520 \pm 0.0640$ & $0.252 \pm 0.0200$ \\
\hline$a(2)=0.250$ & $0.248 \pm 0.0320$ & $0.000 \pm 0.0263$ \\
\hline
\end{tabular}
proximately a shifted version of the true AR model.

Table 1. Two versions of estimated AR parameters

The next simulation result in Fig. 2 shows the locations of estimated poles of the noncausal AR model given by

$$
H_{2}(z)=\frac{1}{\left(1+1.6 z+0.8 z^{2}\right)\left(1-0.6 z^{-1}+0.25 z^{-2}\right)}
$$

where the model order is predetermined as $P=Q=$ 3. Because the order is overestimated, two extra poles

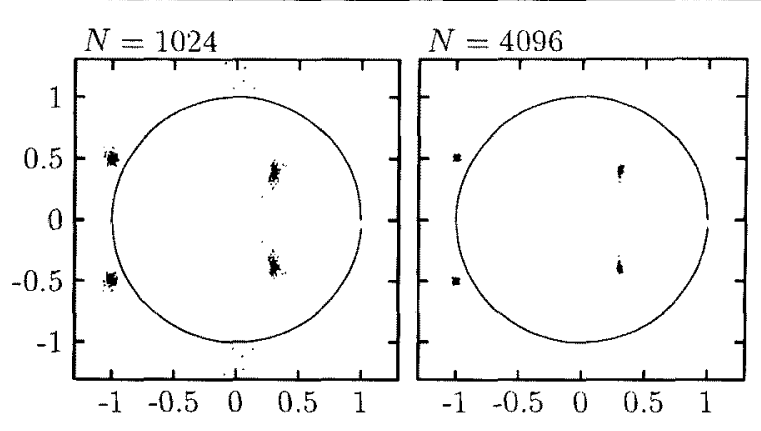

Figure 2 The estimated poles of $H_{2}(z)$

associated with lizear phase factors are introduced and they are located far away from the circle.

\section{Conclusions}

We have presented an approach of identifying noncausal AR model, using 2nd and higher order statistics. Elegant results have been demonstrated by simulatious. Generally, the performance of blind identification using higher order statistics highly depends upon the efficient use of all significant cumulant samples. However the methods in [3][9] exclude the cumulant samples lying in the rectangular regions that are nearly centered on the origin, and then heavily overdetermined equation systems are required in order to obtain acceptable estimates. Fig 1 illustrates the region where the 3rd-order cumulant makes contribution to estimate the AR parameters in our method. This region contains all the most significant samples of 3rd-order cumulant. Thus it can be expected that the algorithm yield better results. Moreover, the order determination often becomes a crucial issue when converting the AR identification to related MA problems. Imprecise system orders may seriously ruin the solutions ${ }^{[3][4]}$. In our method the overestimated order simply leads to equivalent solutions with different linear phases. One may argue that the problem of order determination is converted to that of eigenvector selection. However choosing the proper eigenvector is the final step in the algorithm and does not affect the performance of the estimation. It is superior to those algorithms in which overestimated model order may completely destroy all the computations. Finally, the unique solution is an important issue concerned with using higher order statistics and the unique identification is guaranteed in our method.

Now we briefly discuss the effect of additive Gaussian noise. This issue is also related to whether 2 nd order statistics should be used. Generally the noise affects the estimates of both 2 nd and higher order statistics. Noisy data will introduce bias to 2nd order statistics, 
but higher order statistics can be unbiased. However the deviation of 2nd order statistics are usually much less. That could be one of the reasons why our simulation results show quite small deviation. Thus there is a trade off between the bias and deviation of the estimation. In case of high signal to noise ratio, 2 nd order statistics definitely benefit the estimation. If one is particularly concerned with the biased estimation in lower signal to noise ratio situation, the proposed indirect form of correlation can be applied. In this case relatively large a number of data samples are required in order to obtain reliable estimates of $r(\tau)$ from $C_{3 y}\left(\tau_{1}, \tau_{2}\right)$.

The proposed method is particularly suitable for blind equalization if an adaptive algorithm is developed. This will come to its turn as our further studies. The method can also be easily applied to non-minimum phase MA models, as long as the MA models do not have zeros at $|z|=1$ because this approach essentially requires the systems be (noncausally) reversible.

(Manuscript received Jan.26, 1995; revised Sept.25, 1995)

\section{Reference}

(1) C.L. Nikias, "Bispectrum Estimation: A Digital Signal Processing Framework", Proc. of IEEE, Vol-75, pp. 869-891, Jul. 1987.

(2) J.M. Mendel, "Tutorial on Higher-Order Statistics(Spectra) in Signal Processing and System Theory : Theoretical results and some applications", Proc. of IEEE, Vol-79, 278-305, Mar. 1991

(3) C.L. Nikias and H.H. Chiang, "Higher Order spectrum Estimation Via Noncausal Autoregressive Modeling and Deconvolution", IEEE Trans. Acoust. Speech and Signal Processing, Vol. ASSP-36, pp.1911-1913, Dec. 1988.

(4) G.B. Giannakis and J.M. Mendel, "Identification of Nonminimum Phase Systems Using Higher Order Statistics", IEEE Trans. Acoust. Speech and Signal Processing, Vol. ASSP-37, pp. 361-377, Mar. 1989.

(5) J.A.R. Fonollosa and J. Vidal, "System identification Using a Linear Combination of Cumulants Slices", IEEE Trans. Signal Processing, Vol. SP-41, pp. 24052412, Jul. 1993.

(6) J.K. Tugnait, "Identification of Linear Stochastic Systems via Second- and Fourth-Order Cumulant Matching", IEEE Trans. Info. Theory, Vol. IT-33, pp. 393407, May 1987.

(7) G.B. Giannakis and A. Swami, "On Estimating Noncausal Nonminimum Phase ARMA Models of NonGaussian Processes", IEEE Trans. Acoust. Speech Signal Processing, Vol. ASSP-38, pp.478-495, Mar. 1990.

(8) C.L. Nikias, "ARMA Bispectrum Approach to Nonminimum Phase System Identification", IEEE Trans.
Acoust. Speech and Signal Processing, Vol. ASSP-36, pp.513-525, Apr. 1988.

(9) H.H. Chiang and C.L. Nikias, "Adaptive Deconvolution and Identification of Nonminimum Phase FIR Systems Based on Cumulants", IEEE Trans. Automatic Control, Vol. AC-35, pp. 37-47, Jan. 1990.

Ling CHEN was born on April 13, 1962 in Shaanxi,

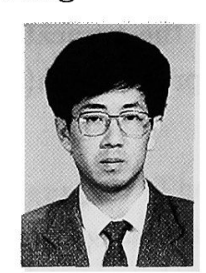
China. He graduated from Xidian University, China, in 1982, and received M.S. degree from Chinese Academy of Science in 1985 and M.E.E. degree from Philips International Institute, the Netherlands in 1990. Now he is a dorctoral candidate at University of Osaka Prefecture.

Hiroji KUSAKA was born in Osaka Prefecture,

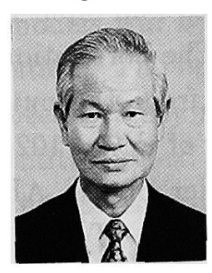
Japan, on February 26, 1933. He received B.E., M.E., and D.E. degrees from University of Osa.ka Prefecture in 1958, 1960 and 1978 respectively. He joined Department of Electrical Engineering, University of Osaka Prefecture and was engaged in research on communications including FDM-FM and CP-FSK communication systems. His current research is focused on spread spectrum communications including digital processing. Now he is a professor of Electronic and Communication Systems. Prof. Kusaka is a senior member of the IEEE

Masanobu KOMINAMI was born on April 14,

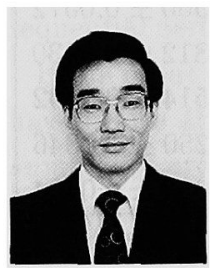
1946. He graduated from University of Osaka Prefecture in 1969. Since he received M.E. degree in 1971, he has joined Department of Electrical Engineering, University of Osaka. Prefecture as an Assistant Professor. His research interests include antenna, microwave and communication systems. He has also received D.E. degree and now he is an associate professor of University of Osaka Prefecture. Prof. Kominami is a member of IEEE.

Qingyie YIN was born in July 1950 in Nanjing, China.

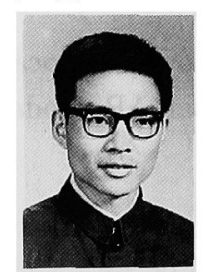
He received B.S., M.S. and Ph.D degrees from Xian Jiaotong University in 1982, 1985 and 1989 respectively. Now he is a professor of Information \& Control Engineering Department at Xian Jiaotong University. His research interests include digital signal processing, neural network and time-frequency analysis. 